\title{
Welcome to the year 2020
}

\author{
Nadia Magnenat-Thalmann ${ }^{1}$
}

(c) Springer-Verlag GmbH Germany, part of Springer Nature 2019

I am very happy to announce that the Visual Computer Journal has progressed significantly during the year 2019 . It has received more than 660 submissions, and the impact factor for 2018 has reached 1.415. We are immensely grateful to our authors who more and more consider the Visual Computer as the journal to publish top results. We take this opportunity to thank our Associate Editors and Reviewers for their tremendous work in reviewing and handling each paper. The journal is very selective as it accepts less than $20 \%$ of the papers submitted.

Throughout 2019, some Associate Editors have left the editorial board of the Visual Computer. I would like to thank them for their great work and contribution:

- Marc Christie, IRISA/INRIA Rennes, France

- Rongrong Ji, Xiamen University, China

- Werner Purgathofer, Vienna University of Technology, Austria

- Zhigeng Pan, Hangzhou Normal University, China

- Adrien Bousseau, INRIA, France

- Didier Stricker, Technische Universitat Kaiserlautern and DFKI, Germany

- Tal Hassner, Open University of Israel and University of Southern California, USA

The editorial board of the Visual Computer is renewed regularly. This year, due to the increase in submissions of papers, we have invited several experts in different fields to join the editorial board. We welcome our new Associate Editors who are listed below:

- Jianbing Shen, Beijing Institute of Technology, China

- Qixiang Ye, University of Chinese Academy of Sciences, China

Nadia Magnenat-Thalmann

thalmann@miralab.ch

1 MIRALab-CUI, University of Geneva, Battelle, Building A, 7, Route de Drize, 1227 Carouge, Geneva, Switzerland
- George Alex Koulieris, Durham University, UK

- Liangliang Cao, UMass Amherst, USA

- Yezhou Yang, Arizona State University, USA

- Robert Sablatnig, TU Wien, Austria

- Marcin Grzegorzek, Universität zu Lübeck, Germany

- Zhou Ren, Wormpex AI Research LLC, USA

- Dong-Ming Yan, National Laboratory of Pattern Recognition (NLPR), Institute of Automation, Chinese Academy of Sciences, China

- Katja Bühler, VRVis Zentrum für Virtual Reality und Visualisierung Forschungs-GmbH, Austria

- Iacopo Masi, University of South California, USA

- Massimiliano Corsini, University of Modena and Reggio Emilia, Italy

- Christoph Garth, TU Kaiserlautern, Germany

- Liang Zhang, Australian National University, Australia

- Ruiping Wang, Institute of Computing Technology, Chinese Academy of Sciences, China

- Yusuf Sahillioğlu, Middle East Technical University, Turkey

- Naser Damer, Fraunhofer IGD, Darmstadt, Germany

- Wangmeng Zuo, Harbin Institute of Technology, China

- Kun Xu, Tsinghua University, China

- Anirban Mukhopadhyay, Technische Universitat Darmstadt, Germany

- Bin Sheng, Shanghai Jiao Tong University, China

- Chunlin Wu, Nankai University, China

- Cornelia M Fermuller, University of Maryland, USA

- Lei Zhang, Beijing Institute of Technology. China

- Xi Peng, Sichuan University, China

- Christian Richardt, University of Bath, UK

- LIAO Jing, City University of Hong Kong, Hong Kong

- Di Lin, Shenzhen University, China

During 2019, the journal published 35 best papers submitted to the Computer Graphics Conference (CGI'2019) that was held in Calgary, Canada in June 2019. During this same year, best papers from other conferences such as ICRIS 2018 and Cyberworlds 2018 were reviewed by our editorial board 
and some of them accepted for publication in the Visual Computer.

Finally, this journal could not exist without the strong support and collaboration of many people. In particular, the two editorial assistants, Dr. Jing Liu and Dr. Manoj Ramanathan our Editorial Director, Rachel Moriarty, our Production Editor, Beate Uhl, and from the journal editorial office, Murugeswari Rangaswamy. I like to thank all of them from for their continuous support and great assistance which is so much needed for a journal that handles continuously so many papers.

Lastly, thanks to all authors who have submitted their work to the Visual Computer as well as to the readers of the Visual Computer. Without them, the journal could not exist.
We hope that 2020 will be a prosperous year for the Visual Computer, and we encourage authors to submit the best of their work.

Nadia Magnenat Thalmann

Editor-in-Chief

The Visual Computer

Publisher's Note Springer Nature remains neutral with regard to jurisdictional claims in published maps and institutional affiliations. 